Sports Training

\title{
Comparison of drop jump force-time profiles of team sport athletes and active controls
}

\author{
Thomas Scarr $^{1}$, Dustin J Oranchuk ${ }^{2,3}$ (D), Daniel Rafferty ${ }^{1}$ \\ ${ }^{1}$ School of Health and Life Sciences, Glasgow Caledonian University, Glasgow, Scotland; ${ }^{2}$ Sports \\ Performance Research Institute New Zealand, Auckland University of Technology, Auckland, New \\ Zealand; ${ }^{3}$ Institute of Health and Sport, Victoria University, Melbourne, Australia.
}

Associate Editor: Ricardo Augusto Barbieri. Estácio UniSEB, Ribeirão Preto, SP, Brazil.

\begin{abstract}
Aim: Lower-body non-contact injuries in team sport athletes (TSAs) are associated when absorbing force, during cutting and landing movements due to a lack of eccentric strength and decreased neuromuscular control leading to excessively higher joint forces. Thus, this project aimed to identify if TSAs had different acceleration and deceleration force profiles compared to a control group (non-TSA) when performing drop jumps (DJs). Methods: University TSAs $(\mathrm{n}=15)$ and non-TSAs $(\mathrm{n}=10)$ performed a series of DJs from a $39 \mathrm{~cm}$ box onto a force-plate. All data were normalized to the individual's body mass. Between-group differences in ground reaction force (GRF), rate of force development (RFD), and propulsive and breaking impulses were compared via t-tests and standardized differences. Results: TSAs had significantly, and meaningfully greater RFD than the non-TSAs ( $p<0.01$, Hedges' $g(E S)=1.24$, $53 \%$ ). While not statistically significant, the non-TSA group produced practically larger mean GRFs than TSAs ( $\mathrm{p}=$ $.09, \mathrm{ES}=0.72,12.1 \%)$. No significant or meaningful between-group differences were detected for propulsive impulse $(\mathrm{p}=0.08, \mathrm{ES}=0.41,9.1 \%)$, braking impulse $(\mathrm{p}=0.85, \mathrm{ES}=0.25,4.6 \%)$, or impulse ratio $(\mathrm{p}=0.35, \mathrm{ES}=0.21,6.7 \%)$. Conclusions: This study shows the presence of significant RFD differences during the DJ in TSAs compared to nonTSAs. Furthermore, this investigation also showed there was no difference between TSA and students in GRF and impulse metrics. Implications from these findings suggest that TSAs can produce force rapidly, but deceleration metrics were not different from untrained students.
\end{abstract}

Keywords: eccentric, force plate, impulse, landing, mechanics, rate of force development.

\section{Introduction}

Understanding injury mechanisms in athletics is vital to reduce the number of injuries in team sports ${ }^{1,2}$. Movements in various sports require deceleration from a sprint or a landing from a jump ${ }^{3}$. Ground reaction forces (GRFs) elicited through the body upon landing have been associated with lower limb injuries ${ }^{4}$. Agel, Aremdt, and Berdshadsky ${ }^{2}$ note that team sport athletes (TSAs) (e.g., soccer, football, basketball, handball) are at high risk for lower limb injuries, including non-contact anterior cruciate ligament tears. This higher injury risk may be due to the landing and cutting required in team sports, combined with dynamic and often unpredictable environments. Research suggests that non-contact injuries in sport are typically correlated with eccentric muscle actions ${ }^{5}$. Hewett, Ford, Hoogenboom, and $\mathrm{Myer}^{3}$ note this can occur due to a combination of low muscular strength (eccentric control), and nervous system disruptions which can lead to excessively high forces imparted onto ligamentous structures.
Movement demands of TSAs are varied, as Beattie et al. $^{6}$, note, TSAs are required to rapidly (sometimes $<250 \mathrm{~ms}$ ) jump, sprint, accelerate, decelerate, cut, and change direction. Similarly, Aagaard et al. ${ }^{7}$, note that the requisite amount of time to develop contractions in sport can be shorter $(<200 \mathrm{~ms})$ than the time required to achieve maximal muscular contraction ( $>300 \mathrm{~ms})$. Due to the necessity of quick contractions, the development, and expression of the rate of force development (RFD) is vital to TSAs. As Tillin et al. ${ }^{8}$, note, RFD is not only important for effective jumping and sprinting performance, but for preventing injuries. The ability to generate very high muscular contractions quickly has an active stability effect on joints ${ }^{9}$. Rapid force creation is important because noncontact knee injuries can occur as quickly as $70 \mathrm{~ms}^{7}$. An effective means of measuring RFD for TSAs is to assess plyometric abilities via force plate technology. To provide more clarity to the kinetics and injuries found in the lower limb, force-plates provide valuable feedback for clinicians and coaches investigating the kinetic characteristics of an individual's movement ${ }^{10}$. 
To better understand athletes' movements, we used the DJ onto a force plate to assess deceleration and acceleration kinetics between TSAs and non-TSAs. There has been some initial research around this topic ${ }^{11-14}$, though methodology and population groups have varied heavily populations tend to be adolescents, recreational athletes ${ }^{11,12}$, female athletes ${ }^{13,14}$, and little research investigating the second landing that follows a maximal vertical jump $^{13,15}$. However, it is vital for sports medicine practitioners, coaches, and athletes to understand GFR and RFD and how they impact sports injuries, training, monitoring, and performance ${ }^{19}$. Therefore, we aimed to characterize the GFRs and RFD of TSAs, and compare them with nonathletic controls (non-TSA). When compared to nonTSAs, we hypothesized that TSAs would demonstrate greater RFDs and lower GRF, likely due to greater muscular strength and coordination.

\section{Methods}

\section{Participants}

Fifteen TSAs $(22.8 \pm 4.2$ years, $82.8 \pm 15.1 \mathrm{~kg}$, $181.4 \pm 12.8 \mathrm{~cm})$, and 10 non-TSAs $(23.7 \pm 0.7$ years, $73.1 \pm 13.4 \mathrm{~kg}, 172.6 \pm 11.4 \mathrm{~cm})$, volunteered to participate. There were no dropouts. Thus, the two groups for this study were 15 TSAs (12 male, 3 female) comprised of rugby ( 3 male), Gaelic Football ( 2 male, 1 female), soccer ( 2 male, 1 female), volleyball (1 male, 1 female) and basketball players (4 male). The non-TSA group had 10 university students (5 female, 5 male). Data collection occurred after an eligibility screening.

TSA's were contacted respective university team sport coaches through email and then scheduled participants for data collection. Posters recruited control group participants with contact details placed around the University and surrounding areas. Eligibility to be included in this study were: 1) a university TSA (basketball, cricket, football/soccer, Gaelic football, ice-hockey, rugby, and volleyball), 2) are 18-35 years old, 3) have no current musculoskeletal injuries, and 4) if they were not a studentathlete, they are a student that has not participated in organized team sports or athletics in the past two years. The Glasgow Caledonian University School of Health and Life Science Ethics Board accepted this protocol, and all participants were briefed on the study procedure before providing written informed consent.

\section{Testing procedures}

Height was measured using a stadiometer (Seca model 213, Chino, California, USA), whereas body mass was measured using an electronic scale (Seca model 813, 136 Chino, California, USA). Participants then performed a standardized warm-up including five minutes on a Monark cycle ergometer (COSMED; Rome, Italy) at a $70 \mathrm{rpm}$, with a $2 \mathrm{~kg}$ resistance, followed by a dynamic warm-up consisting of ten meters of walking lunges, side shuffling, marching, skipping, high knees, as well as ten repetitions of floor-based supine bridges. Participants then were given one minute of rest before being shown a video of individuals partaking the protocol (a side and front view). Participants were allowed up to ten screenings of the tensecond video. Participants were instructed to stand on a box to have their hands on their hips to bring one foot forward, drop down onto the force-plate with two feet and jump "as quickly as possible, maximally as high into the air and then land softly while keeping the hands on the hips". The box was $39 \mathrm{~cm}$ in height, in line with other studies assessing plyometric abilities of team sport athletes $^{15-17}$. Participants were not instructed on how to land. The box was positioned $3 \mathrm{~cm}$ behind the force-plate to allow the participants to step down and slightly forward onto the plate. Participants had up to six practice attempts of the protocol. Between each repetition, participants rested $60 \mathrm{~s}^{18}$. They were prompted to use their practice jumps as submaximal effort. If individuals felt comfortable before six practice jumps, they then partook in the measured DJ protocol. The protocol was identical to the practice jumps, but participants were instructed to jump as quickly off of the plate (as soon as they touched the ground) with maximal intensity and to land "as soft as possible", similar to practice jumps, participants were required to take $60 \mathrm{~s}$ between jumps to ensure adequate rest $^{19}$. Each participant completed a total of six maximal DJs.

\section{Force-plate analysis}

Participants performed the DJ onto a force-plate system (Kistler; model: 9286B, Winterthur, Switzerland) that could measure vertical (Fz) GRF's at $120 \mathrm{~Hz}$ frequency for seven seconds ${ }^{20,21}$. Seven-second recordings were recorded onto the force-plate via Qualisys Track Manager software (QTM Bild 2019.1.4400: Kvarnbergsgatan Sweden). Data in this study analyzed GRF data derived from $\mathrm{Fz}$ on all readings. Data was recorded and imported into a custom Visual 3D software (v6.01.36, C-Motion Inc, Germantown, MD, USA). Data were then processed in Visual 3D with a custom Excel spreadsheet (version 2016; Microsoft Corporation, Redmond, WA, USA). Variables (GRF, RFD, Impulse [propulsive, braking, ratio]) from kinetic data were found, defined, and plotted individually. The impulse ratio was calculated by finding the ratio of the propulsive net impulse to the net braking impulse. All values were reported as absolute values and then created relative to body mass (Normalized - RFD, GRF, and impulse metrics). The vertical force-time metrics were filtered using a Butterworth low-pass filter (with a cut-off frequency of $6 \mathrm{~Hz})^{20}$. An example force-time curve is illustrated in Figure 1. 


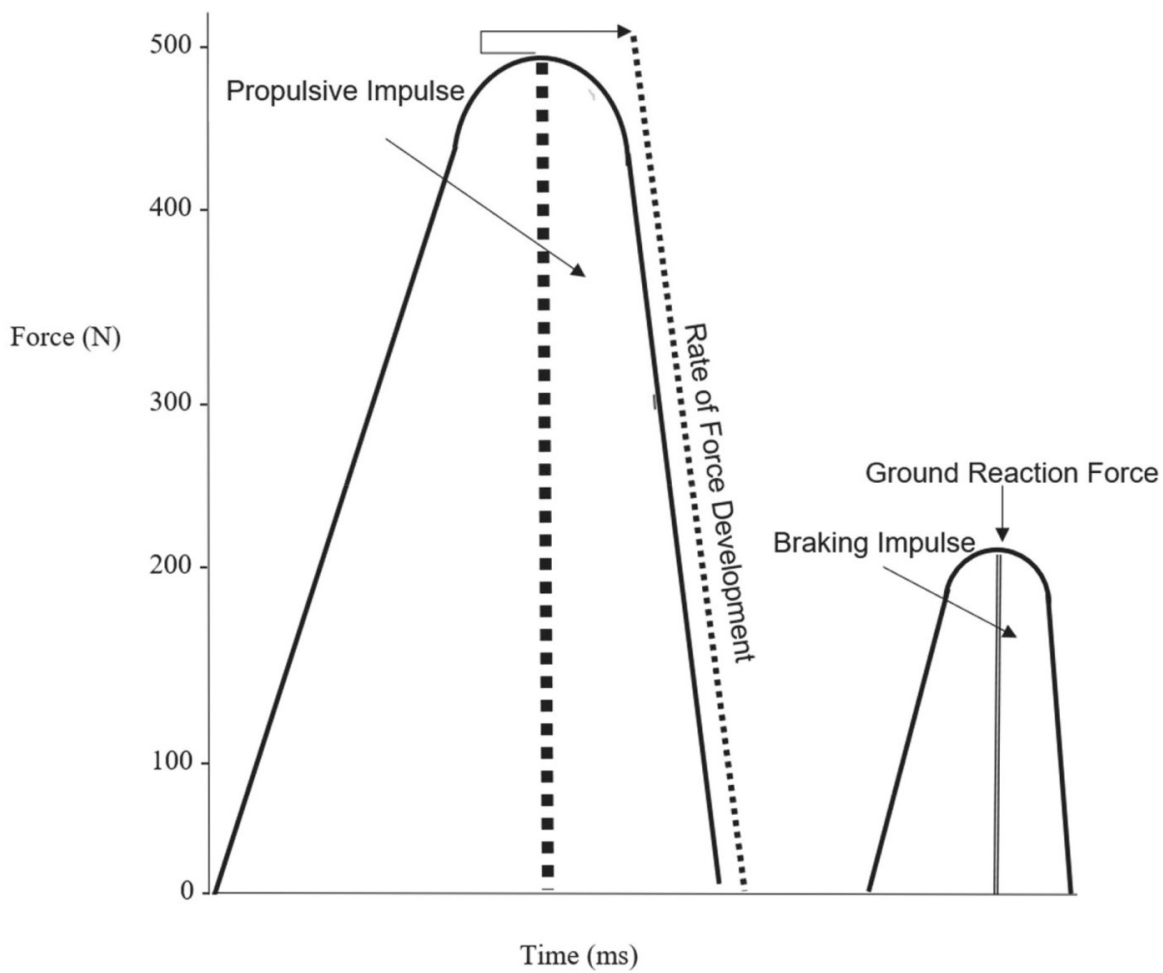

Figure 1 - Illustration of a drop jump force-time curve with examples of how outcome measures were plotted for data collection. The first peak is where the individual lands after stepping off the box. The second peak is the (second) landing after propulsion and was measured as a ground reaction force.

The main outcome measures recorded were GRF $(\mathrm{N})$ and RFD $\left(\mathrm{N} \cdot \mathrm{s}^{-1}\right)$, propulsive net impulse $(\mathrm{N} \cdot \mathrm{s})$, braking net impulse $(\mathrm{N} \cdot \mathrm{s})$, and the impulse ratio (ratio of the propulsive net impulse to braking net impulse). These metrics are clinically relevant as outcome measures are related to acceleration, deceleration, athletic performance and they have an association surrounding athletic injuries ${ }^{22}$. The first GRF was identified as the maximum (peak) value before take-off. The second peak GRF was measured as the maximum value on the second landing phase. RFD was calculated as the $1^{\text {st }}$ peak divided by the time between the $1^{\text {st }}$ peak and the time to leave the plate ${ }^{23}$. The propulsive impulse was calculated as the integral of force from the $1^{\text {st }}$ maximum value until take off from the plate. Braking impulse was calculated as the integral of force from $2^{\text {nd }}$ contact of the plate until the $2^{\text {nd }}$ maximum value was achieved.

\section{Statistical analysis}

Statistical analyses were performed in SPSS version 25 (IBM corporation, Armonk, NY, USA). Normality tests were performed in SPSS to assess the distribution of all outcome measures. Independent t-tests were used to analyze the differences between TSAs and non-TSAs, with the level of significance set at $p<0.05$. Due to the limited sample size, qualitative descriptors of standardized Hedges' $g$ effect sizes (ES) were assessed using these criteria: trivial $<0.2$, small $0.2-0.49$, moderate $0.5-0.79$, large $>0.8^{24}$. An ES of $\geq 0.50$ was considered to be practically important $\mathrm{t}^{25}$. Percent differences are also reported.

\section{Results}

Outcome measures were normally distributed (RFD, GRF, impulse) (Shapiro-Wilk: $p>0.05$ ) aside from impulse ratio (IR) in TSA $(\mathrm{p}=0.009)$. After performing non-parametric tests with independent samples, no between-group differences in impulse ratio were found $(p=0.765)$. No significant between-group differences were found for age, height, or body mass ( $p>0.05)$.

Mean and standard deviation data are presented in Table 1. TSAs had significantly, and meaningfully greater normalized RFD than the non-TSA group $(\mathrm{p}<0.01$, $\mathrm{ES}=1.24,53 \%)$. While not statistically significant, TSAs

Table 1 - Force-plate measurements following a drop jump.

\begin{tabular}{lccccc}
\hline & TSA & non-TSA & p & ES & \% $\boldsymbol{\Delta}$ \\
\hline RFD (N/kg·s $\left.{ }^{-1}\right)$ & $292.1 \pm 105.7$ & $169.7 \pm 75.6$ & $<0.01$ & 1.25 & $53 \%$ \\
GRF (N/kg) & $28.8 \pm 3.1$ & $32.5 \pm 5.9$ & 0.09 & 0.72 & 12.1 \\
PI (N/kg.s) & $5.22 \pm 1.3$ & $5.72 \pm 1.0$ & 0.08 & 0.41 & 9.1 \\
BI (N/kg.s) & $1.50 \pm 0.25$ & $1.57 \pm 0.30$ & 0.85 & 0.25 & 4.6 \\
IR (\%) & $3.73 \pm 1.2$ & $3.99 \pm 1.2$ & 0.35 & 0.21 & 6.7 \\
\hline
\end{tabular}

TSA, team sport athlete; RFD, rate of force development; GRF, ground reaction force; PI, propulsive impulse; BI, breaking impulse; IR, impulse ratio; ES, effect size (Hedges' $g$ ). 
produced larger mean normalized GRFs than the non-TSA group $(\mathrm{p}=0.09, \mathrm{ES}=0.72,12.1 \%)$. No significant or meaningful between-group differences were detected for propulsive impulse $(\mathrm{p}=0.08, \mathrm{ES}=0.41,9.1 \%)$, braking impulse $(\mathrm{p}=0.85, \mathrm{ES}=0.25,4.6 \%)$, or impulse ratio $(\mathrm{p}=0.35, \mathrm{ES}=0.21,6.7 \%)$.

\section{Discussion}

We aimed to identify if TSAs elicited different acceleration and deceleration force profiles compared to non-TSAs. The hypothesis was that TSAs would demonstrate greater RFD with lower GRFs, which was partially supported by our results. The results of the present study suggest that TSAs can produce force at higher rates than non-TSAs but are not any better at decelerating versus non-TSAs, at least when performing the DJ.

The TSAs showed greater concentric RFD compared to the control in the DJ $(\mathrm{p}<0.01, \mathrm{ES}=1.25,53 \%)$. TSAs creating greater RFD than non-trained individuals are consistent throughout the literature, although, methods to support these findings are inconsistent. For example, Tillin, Pain, and Folland ${ }^{26}$ used countermovement jumps (instead of DJ) between rugby players and non-athletes on a force platform to examine GRF profiles. Athletes from this study created forces (absolute RFD) greater than nonathletes $(\mathrm{p}<0.05)$. Tillin et $\mathrm{al}^{8}$, analyzed electromechanical delay and RFD between athletes and non-athletes using an isometric knee extension protocol. Athletes in this investigation displayed twice the RFD during earlyphase RFD compared to untrained participants ${ }^{8}$. However, the results are not easily comparable as the protocol was in an open chain setting compared to a closed/dynamic chain protocol such as the DJ.

Athletes have greater RFD due to several variables. For instance, collegiate TSAs may train and compete upwards of 35 hours per week, where students are often less active ${ }^{27}$. The specific adaptations to imposed demands (SAID) principle could be a contributing factor, as athletes are required to create large forces where less active populations typically do not. Further, if non-TSAs resistancetrained, their ability to create force would likely improve, as lower-body training substantially increases knee-extension $\mathrm{RFD}^{28}$.

Whilst not consistent enough to reach statistical significance, a practically important between-group difference was found for GFR. For example, while nonsignificant $(p=0.09)$, the effect size was large $(E S=0.72$, $12.1 \%$ ). This may be an important finding as previous studies suggest a diminished capacity to attenuate impact during landings is one of the factors related to lower-body injuries $^{29}$; or alternatively, effected by the relatively low number of participants. The literature surrounding GRF's in this chosen population is prevalent, but methodological protocols are inconsistent. For example, Norcross et al. ${ }^{11}$, had 82 participants perform DJs. Participants jumped forward $50 \%$ of their height, down onto a force plate, back up into the air, and landed. The authors found a greater impact on GRFs, noting greater ACL loading. Other studies exist with modified protocols. Podraza and White ${ }^{12}$ had a small sample of students land from a $10.5 \mathrm{~cm}$ box. GRFs decreased where knee moments increased with increased knee flexion upon landing $(p<0.005)$. The authors noted elevated GRFs upon landing in an extended knee position may contribute to non-contact lower-limb injuries ${ }^{12}$.

Due to the lack of comparative studies in the literature, this study fills a specific gap of comparing TSAs to a non-TSA population to understand differences in GRF's in these populations. Whilst interpreting these results, this population did not decelerate more effectively than students but did produce force more rapidly. If athletes are not able to properly decelerate, it is plausible to consider more injuries that could occur due to the correlation between elevated GRFs and lower-body injuries ${ }^{30}$. Further, when an individual's decelerations are uncontrolled and occur too quickly, one's neuromuscular system is put into a position where soft tissue and osseous damage is possible ${ }^{31}$.

There were no significant between-group differences in braking, propulsive, and ratio impulses $(\mathrm{p}=0.08-0.35$, $\mathrm{ES}=0.21-0.41,4.6-9.1 \%)$. Our findings contrast to others in the literature, for example, athletes demonstrate significantly higher values in impulse compared to nonathletes ${ }^{32,33}$. Although, analogous findings to this study were produced by Seegmiller and $\mathrm{McCaw}^{1}$, who utilized a depth drop to assess braking impulse in collegiate gymnasts (and recreational gymnasts) which found no differences. Whilst their protocol was different to ours assessing impulse just during landings from 60 and $90 \mathrm{~cm}$, Milan and Krzysztof ${ }^{34}$, utilized a sample of elite $(\mathrm{n}=6)$ and sub-elite $(\mathrm{n}=6)$ sprinters, who performed countermovement and DJs, and sprint metrics. Disparities in CMJ amid elite and sub-elite were found in jump height, and impulse $(\mathrm{p}<0.05)^{34}$, with analogous findings for the drop jump metrics. Although, athletes in this study were not TSAs which may make this study lack generalizability to our and team sports settings. Evidence around impulse metrics in this area overall contrasts with our findings, which was expected because athletes generally demonstrate greater propulsive and breaking impulses. Perhaps, our sample of athletes could not create impulse as well as the norm.

It is important to discuss the potential effects of jumping and landing mechanics from participants in this study to help understand and interpret the results. This study did not utilize motion capture, but what can be deduced to understand the jumping strategies were the force-plate findings and the protocol itself. While individuals in this study were required to jump with their hands 
on their hips and from a standardized height, the height of the box could have impacted the biomechanical strategies participants used to influence their force plate results. For example, according to Flanagan and Comyns ${ }^{35}$, and Wilson and Flanagan ${ }^{36}$, if an individual's eccentric strength capabilities are not sufficiently strong enough when landing from an elevated height, that may generate higher impact peak forces and create longer loading rates. The participants' jumping technique in our study may have been altered based on their eccentric abilities to land on the first landing (to accept force efficiently) and then quickly create their jump (RFD). Given how TSA created RFD at a significantly higher level than the students, perhaps this was one reason behind their higher RFD.

The jumping technique and force plate data could have been impacted based on the plyometric activity (in general) required and the cues used for the task in this study. For example, individuals were required to "jump as quickly as possible, maximally as high into the air and then to land softly while keeping the hands on the hips". For example, Khuu, Musalem, and Beach ${ }^{37}$ and Young, Wilson, and Byrne ${ }^{38}$ noted that instructing athletes to jump as high and as quickly as possible during drop protocols can impact the jump and landing technique strategies. Specifically, this instruction reduces jump height and increases GRF due to the stiffer landings (with more extended knees). The instructions above are highly relevant to our study and the notion of quickly absorbing and producing force is a major demand in sports requiring stretch-shortening cycle functions (e.g., sprinting, change of direction) $)^{39,40}$.

The findings of the present study may be useful for sports medicine practitioners. Understanding that TSAs elicit higher acceleration metrics but did not absorb force more quickly than untrained individuals provides insight into training programs, rehabilitation programs, and return to play protocols. For example, using GFR analysis throughout the preparatory and in-season periods, and through phases of rehabilitation, may help clinicians dictate training prescription. Further, DJs onto a force-plate could potentially be used as an outcome measure for return to play and monitoring in elite athletics. However, understanding that high relative GRFs and low RFD has been associated with injury mechanisms, these metrics may be useful for sports medicine practitioners.

While the primary goal of the present study was completed, several limitations and directions for future research exist. Firstly, like most sport science studies, our relatively small sample size of 25 requires strong and/or consistent between-group differences to be confident in the outcomes. Therefore, only our RFD result $(\mathrm{p}<0.01$, $\mathrm{ES}=1.25)$ reached sufficient post-hoc power $(1-\beta>0.80)$ to be completely confident in our findings. Additionally, our group sizes were not distributed evenly (15 TSA, 10 non-TSA), and the TSA group was male dominated.
While methods remained consistent, cueing during the DJ may have impacted findings. For example, Winkelman, Palmer, and Ryan $^{41}$, reported differing individual responses to a variety of cues. Thus, language may have impacted how an individual interpreted "jump maximally, quickly, land softly". As the participants in our study were not explicitly instructed on how to jump (knees straight etc.), different between-group jumping styles could have affected our results, instead of other factors, including muscle-tendon-unit stiffness or neural factors. In this study, we only did a drop jump, in future studies, it would be useful to assess athletes to non-athletes via other means such as broad jumps (bilateral and unilateral), lateral bounds, and squat jumps to examine the differences in kinetics seen amongst the groups. Future research may examine bilateral force plates to analyze individuals' lower limb asymmetries and how that may be relevant in accommodating joint forces.

\section{Conclusion}

This investigation displays an approach to evaluate functional lower-body kinetics using the DJ and a forceplate. Further, findings can provide valuable insight into sports medicine practitioners as these metrics play a role in injury, athlete monitoring, and sports performance. This study shows the presence of significant RFD differences during the DJ in TSAs compared to non-TSAs. Furthermore, this investigation also showed there was no difference between TSA and students in GRF and impulse metrics. Implications from these findings suggest that TSAs can produce force rapidly, but deceleration metrics were not different from untrained students. Further, our results demonstrate the non-TSAs may be at a greater risk of injury when beginning a new activity, strengthening the argument for systematic progression of training before full intensity practice or competition for TSAs.

\section{Acknowledgments}

We would like to thank Jane Green, Eric Renaghan, Yannick Plante, Jane McKay, and those who participated in this project.

\section{References}

1. Serner A, Mosler AB, Tol JL, Bahr R, Weir A. Mechanisms of acute adductor longus injuries in male football players: a systematic visual video analysis. Br J Sports Med. 2019;53 (3):158-64.

2. Agel J, Arendt AA, Bershadsky B. Anterior cruciate ligament injury in national athletic association basketball and soccer: a 13-year review. Am J Sports Med. 2005;33 (4):524-30.

3. Hewitt J, Button C, Cronin J, Hume P. Understanding deceleration in sport. Strength Cond J. 2011;33(1):47-52. 
4. Bates NA, Ford KR, Myer GD, Hewett TE. Impact differences in ground reaction force and center of mass between the first and second landing phases of drop jump and their implications for injury risk assessment. J Biomech. 2013;46 (7):1237-41.

5. Reiser RF, Rocheford EC, Armstrong CJ. Building a better understanding of basic mechanical principles through analysis of the vertical jump. Strength Cond J. 2006;28(4):7080 .

6. Beattie K, Carson BP, Lyons M, Kenny IC. The relationship between maximal strength and reactive strength. Int J Sports Physiol Perform. 2017;12(4):548-53.

7. Aagaard P, Simonsen EG, Anderson JL, Magnusson P, Dyhre-Poulsen P. Increased rate of force development and neural drive of human skeletal muscle following resistance training. J Appl Physiol. 2002;93(4):1318-29.

8. Tillin NA, Jimenez-Reyes P, Pain MTG, Folland JP. Neuromuscular performance of explosive power athletes versus untrained individuals. Med Sci Sports Exerc. 2010;42 (4):781-90.

9. Gruber M, Gollhofer A. Impact of sensorimotor on the rate of force development and neural activation. Eur J Appl Physiol. 2004;92(1-2):98-105.

10. Beckham G, Suchomel TJ, Mizuguchi S. Force plate use in performance monitoring and sport science testing. New Stud Athlet. 2014;29(3):25-37.

11. Norcross MF, Lewek MD, Padua DA, Schultz SJ, Weinhold PS, Blackburn JT. Lower extremity energy absorption and biomechanics during landing, part I: Sagittal-plane energy absorption analyses. J Athl Training. 2013;48(6):748-56.

12. Podraza JT, White SC. Effect of knee flexion angle on ground reaction forces, knee moments and muscle co-contraction during an impact-like deceleration landing: implications for the non-contact mechanism of ACL injury. The Knee. 2010;17(4):291-5.

13. Tsai LC, Ko YA, Hammond KE, Xerogeanes JW, Warren GL, Power CM. Increasing hip and knee flexion during a drop-jump task reduces tibiofemoral shear and compressive forces: implications for ACL injury prevention training. $\mathrm{J}$ Sports Sci. 2017;35(24):2405-11.

14. Krosshaug T, Steffen K, Kristianslund E, Nilstad A, Mok $\mathrm{KM}$, Myklebust $\mathrm{G}$, et al. The vertical drop jump is a poor screening test for ACL injuries in female elite soccer and handball players. Am J Sports Med. 2016;44(4):874-83.

15. Chelly MS, Chenem MA, Abid K, Hermassi S, Tabka Z, Shephard RJ. Effects of in-season short-term plyometric training program on leg power, jump-and sprint performance of soccer players. J Strength Cond Res. 2010;24 (10):2670-6.

16. Gehri DJ, Ricard MD, Kleiner DM, Kirkendall DT. A comparison of plyometric training techniques for improving vertical jump ability and energy production. J Strength Cond Res. 1998;12(2):85-9.

17. Thomas K, French D, Hayes PR. The effect of two plyometric training techniques on muscular power and agility in youth soccer players. J Strength Cond Res. 2009;23(1):332-5.

18. Pereira R, Machado M, Santos M, Pereria L. Muscle activation sequence compromises vertical jump performance. Serbian J Sports Sci. 2008;2(3):85-90.
19. Ramirez-Campillo R, Andrade DC, Alvarez C, HenriquezOlguin C, Martinez C, Baez-SanMartin E, et al. The effects of interset rest on adaptation to 7 weeks of explosive training in young soccer players. J Sports Sci Med. 2014;13 (2):287-96.

20. Fransz DP, Huurnink A, de Boode V, Kingma I, van Dieen JH. Time to stabilization in single leg drop jump landings: An examination of calculation methods and assessment of differences in sample rate, filter settings and trial length on outcome values. Gait Posture. 2016;4(1):63-9.

21. Hori N, Newton RU, Kawamori N, McGuigan MR, Kraemer WJ, Nosaka K. Reliability of performance measurements derived From ground reaction force data during countermovement jump and the influence of sampling frequency. J Strength Cond Res. 2009;23(3):874-82.

22. Angelozzi M, Madama M, Corsica C, Calvisi V, Properzi G, McCaw ST, et al. Rate of force development as an adjunctive outcome measure for return-to-sport decisions after anterior cruciate ligament reconstruction. J Orthop Sports Phys Ther. 2012;42(9):772-80.

23. Haff GG, Ruben RP, Lider J, Twine C, Cormie P. A comparison of methods for determining the rate of force development during isometric midthigh clean pulls. J Strength Cond Res. 2015;29(2):386-95.

24. Fritz CO, Morris PE, Richler JJ. Effect size estimates: current use, calculations, and interpretation. J Exp Psychol Gen. 2012;141(1):2-18.

25. Oranchuk DJ, Robinson TL, Switaj ZJ, Drinkwater EJ. Comparison of the hang high-pull and loaded jump squat for the development of vertical jump and isometric force-time characteristics. J Strength Cond Res. 2019;33(1):17-24.

26. Tillin NA, Pain MT, Folland JP. Explosive force production during isometric squats correlates with athletic performance in rugby union players. J Sports Sci. 2013;31(1):66-76.

27. National Collegiate Athletic Association. Goals and score studies. Available from: https://www.ncaa.org/sites/default/ files/2017GOALS_Time_demands_20170628.pdf [Accessed 20th June 2018].

28. Anderson LL, Anderson JL, Zebis MK, Aagaard P. Early and late rate of force development: Differential adaptive responses to resistance training? Scand J Med Sci Sports. 2010;20(1):162-9.

29. Chappell JD, Herman DC, Knight BS, Kirkendall DT, Garrett WE, Yu B. Effect of fatigue on knee kinetics and kinematics in stop-jump tasks. Am J Sports Med. 2005;33 (7):1022-9.

30. Delfico AJ, Garret WE. Mechanisms of injury of the anterior cruciate ligament in soccer players. Clin Sports Med. 1998;17(4):779-85.

31. Hewett TE, Ford KR, Hoogenboom BJ, Myer GD. Understanding and preventing ACL injuries: current biomechanical and epidemiologic considerations - update 2010. North Am J Sports Phys Ther. 2010;5(4):234.

32. Cormie P, McBride JM, McCaulley GO. Power-time, forcetime and velocity-time curve analysis of the countermovement jump: Impact of training. J Strength Cond Res. 2009;23(1):177-86. 
33. Seegmiller JG, McCaw ST. Ground reaction forces among gymnasts and recreational athletes in drop landings. J Athl Train. 2003;38(4):311-4.

34. Milan C, Krzysztof M. Differences between the elite and sub-elite sprinters in kinematic and dynamic determinations of countermovement jump and drop jump. J Strength Cond Res. 2013;27(11):3021-7.

35. Flanagan EP, Comyns T. The use of contract time and the reactive strength index to optimize fast stretch-shortening cycle training. Strength Cond J. 2008;30(5):32-8.

36. Wilson JM, Flanagan EP. The role of elastic energy in activities with high force and power requirements: a brief review. J Strength Cond Res. 2008;22(5):1705-15.

37. Khuu S, Musalem LL, Beach TAC. Verbal instructions acutely affect drop jump vertical jump biomechanics-Implications for athletic performance and injury risk assessments. J Strength Cond Res. 2015;29(10):2816-26.

38. Young WB, Wilson GJ, Byrne C. A comparison of drop jump training methods: effects on leg extensor strength qualities and jumping performance. Int J Sports Med. 1999;20 (5):295-303.

39. Marchall BM, Moran KA. Which drop jump technique is most effective at enhancing countermovement jump ability, "countermovement" drop jump or "bounce" drop jump? J Sports Sci. 2013;31(12):1368-74.
40. Bobbert MF, Huijing PA, van Ingen Schenau GJ. Drop jumping. II. The influence of dropping height on the biomechanics of drop jumping. Med Sci Sports Exerc. 1987;19 (4):339-46.

41. Winkelman N, Palmer K, Ryan L. Experience level influences the effect of attentional focus on sprint performance. Hum Movement Sci. 2017;52:84-95.

\section{Corresponding Author}

Dustin J. Oranchuk. AUT-Millennium, 17 Antares Place, Mairangi Bay, Auckland, New Zealand.

E-mail: dustinoranchuk@gmail.com.

Manuscript received on March 14, 2021

Manuscript accepted on August 2, 2021

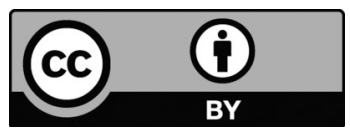

Motriz. The Journal of Physical Education. UNESP. Rio Claro, SP, Brazil - eISSN: 1980-6574 - under a license Creative Commons - Version 4.0 